\title{
A competing endogenous RNA mechanism in glioblastoma is investigated by bioinformatics analysis
}

\section{Jinsheng Wang}

Shangdong Provincial

\section{Yutao Wang}

Shangdong Provincial Key Laboratory of Test Technology on Food Quality and Safety

Lei Gao ( $\sim$ herescience@outlook.com )

Shangdong Provincial Key Laboratory of Test Technology on Food Quality and Safety https://orcid.org/0000-0002-9328-6014

\section{Yuhua Zhao}

Shangdong Provincial Key Laboratory of Test Technology on Food Quality and Safety Junhua Liu

Shangdong Provincial Key Laboratory of Test Technology on Food Quality and Safety

\section{Da Cai}

Shangdong Provincial Key Laboratory of Test Technology on Food Quality and Safety

\section{Xiao Zhang}

Shangdong Provincial Key Laboratory of Test Technology on Food Quality and Safety

\section{Research article}

Keywords: glioblastoma, SEMA4D, ceRNA, bioinformatics analysis

Posted Date: January 17th, 2020

DOI: https://doi.org/10.21203/rs.2.21101/v1

License: (c) (1) This work is licensed under a Creative Commons Attribution 4.0 International License. Read Full License 


\section{Abstract}

\section{Background}

Glioblastoma (GBM) is the most aggressive and most lethal primary malignant brain tumor, the 5-year survival rate of which is less than $5 \%$. Novel potential molecular and mechanism of GBM need to investigate.

Materials and methods

Microarray data of GSE15824 was downloaded from GEO. Differentially expressed genes and IncRNAs were screened by Limma package in R studio, and pathway enrichment analysis was performed by clusterprofiler package in R studio and IPA. The ceRNA mechanism was analyzed and predicted by several kinds of online public databases.

Results

There were 567 differentially expressed genes and 121 differentially expressed IncRNAs in GBM. And differentially expressed genes were mainly enriched in Tuberculosis, Staphylococcus aureus infection, Systemic lupus erythematosus, Basal cell carcinoma, TGF-beta signaling pathway and p53 signaling pathway. Besides, Neuroinflammation signaling pathway, Role of NFAT in regulation of the immune response, and Dendritic cell maturation were significantly activated in GBM. According to the analysis of target miRNAs of SEM4D and OSER1-AS1, a possible ceRNA mechanism OSER1-AS1/hsa-miR520h/SEMA4D axis was predicted in GBM.

\section{Conclusion}

Bioinformatics analysis was employed to analyze GSE15824 chip, and predict the potential mechanism. The results revealed that the ceRNA mechanism, OSER1-AS1/hsa-miR-520h/SEMA4D axis, might play a vital role in GBM.

\section{Background}

Glioblastoma (GBM) is the most aggressive and most lethal primary malignant brain tumor in central nervous system (1), which is characterized by genetic and epigenetic variation among tumor cells (2). GBM has three transcriptional subtypes: proneural (PN), classical, and mesenchymal (MES), among which, PN cells are considered as the precursor of GBM (3). At present, radiotherapy and chemotherapy are the mainly treatments for GBM, targeting proliferating tumor cells and inducing effective toxic side effects by damaging normal proliferating cells (4). In addition, according to the ability of surgeons to distinguish cancerous and non-cancerous tissues, extended resection without neurological deficits after surgery is still a vital component to improve the prognosis of patients (5). However, due to the histological heterogeneity, GBM can't be completely eradicated, resulting in recurrence (5). Because of the blood-brain barrier (BBB), the drug accessibility is extremely low (6). Moreover, the increase of genomic heterogeneity 
caused by new mutations in tumor results in the development of drug resistance, which restricts the longterm efficacy of treatment (7). The average survival time of GBM is only 15 months (1), and the 5-year survival rate is less than $5 \%(8)$. Therefore, it is necessary to investigate molecule target and potential molecular mechanism of GBM and develop novel treatment strategies.

Semaphorins are an extended and various family of secretory or membrane-related glycoproteins defined by the domain of cysteine-rich semaphorin proteins, which were first recognized in the central nervous system and found to act as the repulsive guidance molecules of axon (9). However, recent researches have shown that semaphorins regulate not only the morphology of cells, but also the functions of cell division, proliferation and differentiation (10). Semaphorin4D (SEMA4D), which is originally described as CD100 antigen in immune cells (11), is a glycoprotein belonging to the class IV of semaphorin family, and can exist in secreted or membrane-bound form (12). Besides, SEMA4D is also the first member of semaphorin family to be found to have immunomodulatory activity (11). There are currently 3 cellular receptors identified as SEMA4D: plexin B1 (PLXNB1), plexin B2 (PLXNB2), and CD72 (13). SEMA4D binding to PLXNB1, a high affinity (1 nM) SEMA4D receptor, induces endothelial cell migration and activation, and promotes tumor cell migration $(14,15)$. Besides, it also induces neuronal growth cone collapse, neural precursor cell apoptosis and processes of oligodendrocytes extension collapse and apoptosis (16-18). PLXNB2 is an intermediate affinity receptor of SEMA4D, which mainly binds to SEMA4C and is considered to participate in the repair of epidermal wounds (19). CD72 binds to SEMA4D with low affinity $(K D=300 \mathrm{nM})$, which seems to serve as a negative regulator of $B$ cell response by tyrosine phosphatase SHP-1 $(20,21)$. Studies have shown that SEMA4D is highly expressed not only in the most solid tumor cell membranes and tumor-associated macrophages (TAMs) such as breast, prostate, and colon, but also in tumor stroma (22). However, few researches have been conducted on the role of SEMA4D in GBM, the effects of SEMA4D on GBM are unclear, and the molecular mechanism is unstudied.

Based on the Human Genome Project and the emergence of high-throughput genome technology, biomedical research in the past two decades has produced plenty of diverse biological datasets (23). Gene expression profiling building on microarray is widely used in cancer research to authenticate biomarkers that may be contributed to clinical diagnosis, prognosis or treatment response (24). In this study, the data from GSE15824 was subjected to differential expression analysis, pathway enrichment analysis, and the correlation analysis between mRNAs and mRNAs or mRNAs and IncRNAs. In addition, built on the public databases, OSER1-AS1/hsa-miR-520 h/SEMA4D was predicted to be the possible competing endogenous RNAs (ceRNA) mechanism in GBM.

\section{Materials And Methods}

\section{Microarray analysis}

The data of GSE15824 chip, which based on the platform GPL570 (Affymetrix Human Genome U133 Plus 2.0 Array) were downloaded from Gene Expression Omnibus (GEO) database, including 45 samples. 
12 primary GBM samples as the tumor group and 3 normal human astrocytes as the control group were analyzed in this study.

\section{Screening of differentially expressed genes and IncRNAs}

The differentially expressed genes and IncRNAs were conducted by using the Limma package in R studio. After the data were normalized, the clustering analysis was performed and the genes or IncRNAs with differential expression were screened. The differentially expressed genes were screened under the standard $\mid \log _{10}$ Fold Change| $>6$, adjust $P$ value $<0.0 .5$, and the differentially expressed IncRNAs were screened under the standard $\mid \log _{10}$ Fold Changel $>2$, adjust $P$ value $<0.0 .5$.

\section{Pathway enrichment analysis}

The Gene Set Enrichment Analysis (GSEA) was performed built on the gene set of Kyoto Encyclopedia of Genes and Genomes (KEGG) (gseKEGG) by the clusterprofiler package of R studio. The threshold values were set as $P<0.05$ and counts $>2$.

\section{Ingenuity Pathway Analysis}

The Ingenuity Pathway Analysis (IPA) software was used to analyze the distribution of differentially expressed genes in classical pathways. Z-score $>0$ indicated that the pathway was activated in GBM, whereas Z-score $<0$ suggested that the pathway was inhibited. $\mid Z$ - score| $>2$ is considered statistically significant. In addition, the mRNA-mRNA correlation network was also obtained by IPA.

\section{ceRNA mechanism prediction}

The ceRNA mechanism was analyzed and predicted by several kinds of online public databases. The miRNAs targeted mRNA SEMA4D were predicted by Starbase, miRmap and miRTarbase database, and then the online tool Venny was employed to screen out miRNAs contained in these three database. The miRNAs targeting IncRNA OSER1-AS1 were predicted by Starbase. Finally, the miRNA that targeted SEMA4D as well as OSER1-AS1 was also screened by Venny.

\section{Results}

\section{The expression profile of differential genes and IncRNAs in GBM.}

For purpose of identifying genes that may plan a vital role in GBM, the chip GSE15824 from GEO database was analyzed by using R studio. Limma package was employed to analyze differentially genes and IncRNAs. All the differentially expressed genes in GBM were presented in Figure 1A and Supplementary table 1, including 369 upregulated genes and 198 downregulated genes, and the top 421435 differentially expressed genes were shown in Figure 1B. It was found that SEMA4D was highly expressed in GBM. Besides, there were 121 differentially expressed IncRNAs in GBM (Figure 2A and 
Supplementary table 2), among which 46 were highly expressed and 65 were poorly expressed, and Figure 2B showed the top 1-20 of differentially expressed IncRNAs.

\section{Pathway enrichment based on gesKEGG was performed.}

Pathway enrichment was performed by the clusterprofiler package in R studio, and the top 8 activated and top 8 suppressed signaling pathways were presented in Figure 3A. The results revealed that differentially expressed genes were mainly enriched in Tuberculosis, Staphylococcus aureus infection, Systemic lupus erythematosus, Basal cell carcinoma, TGF-beta signaling pathway and p53 signaling pathway. In addition, not only was the correlation between enrichment signaling pathways evaluated, as shown in Figure 3B, but also the correlation between enrichment signaling pathways and differentially expressed genes was analyzed, as shown in Figure 3C. Furthermore, the results in Figure $\mathbf{3 C}$ indicated that upregulated genes were mainly contributed in Tuberculosis, Staphylococcus aureus infection, Systemic lupus erythematosus, Neuroactive ligand-receptor interaction, Renin secretion, Alzheimer disease, Amyotrophic lateral sclerosis (ALS) and Vasopressin-regulated water reabsorption, while the downregulated genes were mainly involved in TGF-beta signaling pathway and Basal cell carcinoma.

\section{The molecular correlation network about SEMA4D.}

Ingenuity Pathway Analysis (IPA) software was employed to demonstrate the significant enrichment of differentially expressed genes in canonical pathways, the results of which suggested that Neuroinflammation signaling pathway, Role of NFAT in regulation of the immune response, and Dendritic cell maturation were significantly activated in GBM (Figure 4A). The correlation between SEMA4D and differentially expressed genes was also analyzed by IPA. It was found that SEMA4D might affect more genes by acting on genes PTPRC, HLA-DQB1 and CCL2 (Figure 4B). Moreover, the co-expression network of SEMA4D and differentially expressed IncRNAs was analyzed and visualized by the Cytoscape software (Figure 4C). 10 upregulated IncRNAs in GBM were demonstrated to be correlated to SEMA4D.

\section{Prediction of possible ceRNA mechanisms related to SEMA4D in GBM.}

In order to investigate the possible ceRNA mechanisms related to SEMA4D in GBM, the miRNAs targeting SEMA4D were predicted using Starbase, miRmap and miRTarbase public databases. There were 135 miRNAs targeting SEMA4D in Starbase, 1025 in miRmap and 26 in miRTarbase. However, only 4 miRNAs (hsa-miR-381-3p, hsa-miR-335-5p, hsa-miR-520h and hsa-miR-300) were overlapped in 3 databases analyzing by Venny (Figure 5A). Combined with the miRNAs targeting IncRNA OSER1-AS1 in Starbase (Supplementary table 3), it was found that hsa-miR-520h not only targeted SEMA4D, but also was a target miRNA of IncRNA OSER1-AS1. What's more, the target sites between OSER1-AS1 and hsa-miR520h, or hsa-miR-520h and SEMA4D were predicted by Starbase (Figure 5B). All of the results indicated that OSER1-AS1/hsa-miR-520h/SEMA4D axis was a possible ceRNA mechanism in GBM.

\section{Discussion}


Glioblastoma (GBM) is the most common aggressive primary brain tumor originating from the central nervous system (CNS) and also one of the deadliest malignancies (25). The current treatment of GBM includes a combination of surgery, radiotherapy and chemotherapy, and the standard chemotherapy drug in use is temozolomide (TMZ). Nevertheless, on account of the high drug resistance rate to treatment and recurrence rate of GBM patients, the overall survival is poor, with only a median survival period of about 15 months (26). Hence, the novel therapeutic target and treatment strategy should be investigated.

In the present study, the data from GSE15824 building on GEO were employed to analyze the significant differential gene expression profiles and differentially expressed IncRNAs by Limma package in R. SEMA4D, a member of semaphorin family, was demonstrated to be a significantly upregulated gene in GBM. There were already some researches that reported that SEMA4D was upregulated in multiple types of cancer, including colon, prostate, breast, head, and neck carcinomas (27). Studies by John R. Basile et al. revealed that the size and vascular distribution of head and neck squamous cell carcinomas (HNSCC) tumor xenografts could be significantly reduced after SEMA4D knockdown (28). Besides, SEMA4D also promoted the cytotoxic activation of microglia and suppressed functional recovery after cerebral ischemia (29). Moreover, SEMA4D damaged the integrity of blood-brain barrier and promoted inflammatory response by binding to PlexinB1 after transient middle cerebral artery occlusion (30). Yun Zhang et al. indicated that SEMA4D was highly expressed in NK cell line, NK92, and the interaction between SEMA4D and Plexin-B1 played a crucial part in NK cell-mediated cytotoxicity against glioma cells (31). However, the further mechanism of SEMA4D in GBM has not been reported.

The signaling pathways enrichment based on GSEA in the present study suggested that genes upregulated in GBM were mainly contributed in Tuberculosis, Staphylococcus aureus infection, Systemic lupus erythematosus, Neuroactive ligand-receptor interaction, Renin secretion, Alzheimer disease, Amyotrophic lateral sclerosis (ALS) and Vasopressin-regulated water reabsorption, while the downregulated genes were major in TGF-beta signaling pathway and Basal cell carcinoma. IPA software was employed to demonstrate the significant enrichment of differentially expressed genes in canonical pathways, the results of which suggested that Neuroinflammation signaling pathway, Role of NFAT in regulation of the immune response, and Dendritic cell maturation were significantly activated in GBM. Guanlin Li et al. demonstrated that the crosstalk between SEMA4D and Met could activate Met signaling and promote the invasion and differentiation of trophoblasts (32). Furthermore, SEMA4D could activated the RhoA/ROCK pathway, and the modulation of SEMA4D/PulnB1 blocked tumor blood supply through vasulogenic mimicry formation, which ultimately inhibited the growth and metastasis of non-small cell lung cancer (NSCLC) (27). There was also one report indicating that SEMA4D promoted cell proliferation and metastasis in bladder cancer by activating the PI3K/AKT pathway (33).

The RNA-mRNA correlation network indicated that SEMA4D was at the center position, which suggested that SEMA4D might play an important role in GBM by regulating other genes. In addition, the mRNAIncRNA correlation network revealed that there were some differentially expressed IncRNAs in GBM which were positively or negatively correlated with SEMA4D expression, and OSER1-AS1 was one of the upregulated IncRNAs. LncRNAs (long noncoding RNAs) are a kind of ncRNAs with more than 200 
nucleotides in length, which have no coding ability for proteins (34). LncRNAs can regulate gene expression either in cis or in trans by directly impacting the sequences of the promoters or enhancers of target genes, or through influencing long-distance genes with other helper molecules (35). Besides, IncRNAs can also impress the protein concentrations produced by target genes partly through affecting the stability or the translational efficiency of mRNA (36). There was a study that confirmed that IncRNA OSER1-AS1acted as a ceRNA to promote hepatocellular carcinoma progression by regulating miR3723p/Rab23 axis (37). The ceRNA (competing endogenous RNA) has been proposed and studied in recent years. Tay et al. indicated that in the hypothesis on ceRNA, diverse molecular, such as IncRNAs, circRNAs, and mRNAs competed to bind to a specific molecular target, miRNA (microRNA), thus modulating miRNA activity (38). miRNAs were other types ncRNAs, which were highly conserved among species, regulate gene expression through binding to the $3^{\prime}$-untranslated region of target mRNAs and play central roles in the ceRNA hypothesis (39). Qixue Wang et al. has indicated in their research that IncRNAs competed with target mRNA to bind miRNA, or served as "miRNA sponges", which indirectly suppressed the regulation of miRNAs on their target mRNA, thus impacting the biological behaviors of tumor cells (40). By analyzing the prediction results of miRNAs targeting SEMA4D built on Starbase, miRmap, and miRTarbase public database, it was found that there were 4 co-miRNAs: hsa-miR-381-3p, hsa-miR-335-5p, hsa-miR-520 $\mathrm{h}$ and hsa-miR-300. Combined with the prediction results of IncRNA-target miRNAs, we proposed a possible ceRNA mechanism, that is, OSER1-AS1/hsa-miR-520 h/SEMA4D, which might play a vital role in the progression of GBM.

\section{Conclusions}

In summary, the data from GSE15824 was subjected to bioinformatics analysis, and the correlation analysis between mRNAs and mRNAs or mRNAs and IncRNAs was performed built on the public databases. OSER1-AS1/hsa-miR-520 h/SEMA4D was predicted to be the possible ceRNA mechanism in GBM. However, the specific effects of SEMA4D on GBM should be further investigated in vitro and vivo. Besides, the ceRNA mechanism of OSER1-AS1/h-miR-520 h/SEMA4D axis should be confirmed in GBM.

\section{Declarations}

\section{Ethics approval and consent to participate}

Not applicable.

\section{Consent for publication}

Not applicable.

\section{Availability of data and materials}

Not applicable. 


\section{Competing interests}

The authors declare that they have no competing interests.

\section{Funding}

This work was supported by the key research and development plan of Shandong Province "Application of 3D printing model of colon cancer in vitro in targeted therapy" (N0.2017G006007). This work was supported by the key research and development plan of Shandong Province "Study on the role of free SEMA4D, a new target of ani-tumor angiogenesis, in colon cancer" (NO.2017GSF221018).

\section{Authors' contributions}

JW put forward the concept and design, which made substantial contributions to this study. JW and DC performed all the experiments involved in the present research. $L G$ and $X Z$ collected and analyzed the data. JW, YZ and JL produced this manuscript. YW reviewed analysis results and revised the manuscript. All authors read and approved the final manuscript.

\section{Acknowledgments}

This work was supported by the key research and development plan of Shandong Province "Application of 3D printing model of colon cancer in vitro in targeted therapy". This work was supported by the key research and development plan of Shandong Province "Study on the role of free SEMA4D, a new target of ani-tumor angiogenesis, in colon cancer".

\section{References}

1. J.H. Lee, J.E. Lee, J.Y. Kahng, S.H. Kim, J.S. Park, S.J. Yoon, et al. Human glioblastoma arises from subventricular zone cells with low-level driver mutations. Nature. 2018, 560(7717): 243-247.

2. M.L. Broekman, S.L.N. Maas, E.R. Abels, T.R. Mempel, A.M. Krichevsky and X.O. Breakefield. Multidimensional communication in the microenvirons of glioblastoma. Nat Rev Neurol. 2018, 14(8): 482-495.

3. M.S. Pavlyukov, H. Yu, S. Bastola, M. Minata, V.O. Shender, Y. Lee, et al. Apoptotic Cell-Derived Extracellular Vesicles Promote Malignancy of Glioblastoma Via Intercellular Transfer of Splicing Factors. Cancer Cell. 2018, 34(1): 119-135. e10.

4. Y. Shi, S.K. Lim, Q. Liang, S.V. lyer, H.Y. Wang, Z. Wang, et al. Gboxin is an oxidative phosphorylation inhibitor that targets glioblastoma. Nature. 2019, 567(7748): 341-346.

5. S. Pinel, N. Thomas, C. Boura and M. Barberi-Heyob. Approaches to physical stimulation of metallic nanoparticles for glioblastoma treatment. Adv Drug Deliv Rev. 2019, 138: 344-357.

6. Y. Jiang, W. Yang, J. Zhang, F. Meng and Z. Zhong. Protein Toxin Chaperoned by LRP-1-Targeted Virus-Mimicking Vesicles Induces High-Efficiency Glioblastoma Therapy In Vivo. Adv Mater. 2018, 30(30): e1800316. 
7. R.B. Puchalski, N. Shah, J. Miller, R. Dalley, S.R. Nomura, J.G. Yoon, et al. An anatomic transcriptional atlas of human glioblastoma. Science. 2018, 360(6389): 660-663.

8. E.S. Polson, V.B. Kuchler, C. Abbosh, E.M. Ross, R.K. Mathew, H.A. Beard, et al. KHS101 disrupts energy metabolism in human glioblastoma cells and reduces tumor growth in mice. Sci Transl Med. 2018, 10(454).

9. A. Bulloj, A. Maminishkis, M. Mizui and S.C. Finnemann. Semaphorin4D-PlexinB1 Signaling Attenuates Photoreceptor Outer Segment Phagocytosis by Reducing Rac1 Activity of RPE Cells. Mol Neurobiol. 2018, 55(5): 4320-4332.

10. K. Otsu, H. Ida-Yonemochi, N. Fujiwara and H. Harada. The Semaphorin 4D-RhoA-Akt Signal Cascade Regulates Enamel Matrix Secretion in Coordination With Cell Polarization During Ameloblast Differentiation. J Bone Miner Res. 2016, 31(11): 1943-1954.

11. L. Tamagnone and G. Franzolin. Targeting Semaphorin 4D in Cancer: A Look from Different Perspectives. Cancer Res. 2019, 79(20): 5146-5148.

12. R. Derakhshandeh, S. Sanadhya, K. Lee Han, H. Chen, O. Goloubeva, T.J. Webb, et al. Semaphorin 4D in human head and neck cancer tissue and peripheral blood: A dense fibrotic peri-tumoral stromal phenotype. Oncotarget. 2018, 9(13): 11126-11144.

13. S. Shen, Y. Ke, E. Dang, H. Fang, Y. Chang, J. Zhang, et al. Semaphorin 4D from CD15(+) Granulocytes via ADAM10-Induced Cleavage Contributes to Antibody Production in Bullous Pemphigoid. J Invest Dermatol. 2018, 138(3): 588-597.

14. J.R. Basile, A. Barac, T. Zhu, K.L. Guan and J.S. Gutkind. Class IV semaphorins promote angiogenesis by stimulating Rho-initiated pathways through plexin-B. Cancer Res. 2004, 64(15): 5212-24.

15. S. Giordano, S. Corso, P. Conrotto, S. Artigiani, G. Gilestro, D. Barberis, et al. The semaphorin 4D receptor controls invasive growth by coupling with Met. Nat Cell Biol. 2002, 4(9): 720-4.

16. P. Giraudon, P. Vincent, C. Vuaillat, O. Verlaeten, L. Cartier, A. Marie-Cardine, et al. Semaphorin CD100 from activated $T$ lymphocytes induces process extension collapse in oligodendrocytes and death of immature neural cells. J Immunol. 2004, 172(2): 1246-55.

17. B. Niederost, T. Oertle, J. Fritsche, R.A. McKinney and C.E. Bandtlow. Nogo-A and myelin-associated glycoprotein mediate neurite growth inhibition by antagonistic regulation of RhoA and Rac1. J Neurosci. 2002, 22(23): 10368-76.

18. X. Liang, N.A. Draghi and M.D. Resh. Signaling from integrins to Fyn to Rho family GTPases regulates morphologic differentiation of oligodendrocytes. J Neurosci. 2004, 24(32): 7140-9.

19. D.A. Witherden, M. Watanabe, O. Garijo, S.E. Rieder, G. Sarkisyan, S.J. Cronin, et al. The CD100 receptor interacts with its plexin $\mathrm{B} 2$ ligand to regulate epidermal gammadelta $\mathrm{T}$ cell function. Immunity. 2012, 37(2): 314-25.

20. A. Kumanogoh and H. Kikutani. The CD100-CD72 interaction: a novel mechanism of immune regulation. Trends Immunol. 2001, 22(12): 670-6.

21. J.E. Leonard, T.L. Fisher, L.A. Winter, C.A. Cornelius, C. Reilly, E.S. Smith, et al. Nonclinical Safety Evaluation of VX15/2503, a Humanized IgG4 Anti-SEMA4D Antibody. Mol Cancer Ther. 2015, 14(4): 
964-72.

22. I. Zuazo-Gaztelu, M. Paez-Ribes, P. Carrasco, L. Martin, A. Soler, M. Martinez-Lozano, et al. Antitumor Effects of Anti-Semaphorin 4D Antibody Unravel a Novel Proinvasive Mechanism of VascularTargeting Agents. Cancer Res. 2019, 79(20): 5328-5341.

23. K. Qu, S. Garamszegi, F. Wu, H. Thorvaldsdottir, T. Liefeld, M. Ocana, et al. Integrative genomic analysis by interoperation of bioinformatics tools in GenomeSpace. Nat Methods. 2016, 13(3): 245247.

24. W. Zhang, Y. Yu, F. Hertwig, J. Thierry-Mieg, W. Zhang, D. Thierry-Mieg, et al. Comparison of RNA-seq and microarray-based models for clinical endpoint prediction. Genome Biol. 2015, 16(1): 133.

25. D. Patel, F. Ahmad, D.M. Kambach, Q. Sun, A.S. Halim, T. Kramp, et al. LXRbeta controls glioblastoma cell growth, lipid balance, and immune modulation independently of ABCA1. Sci Rep. 2019, 9(1): 15458.

26. J. Muller Bark, A. Kulasinghe, B. Chua, B.W. Day and C. Punyadeera. Circulating biomarkers in patients with glioblastoma. Br J Cancer. 2019, doi: 10.1038/s41416-019-0603-6.

27. Y. Xia, X.Y. Cai, J.Q. Fan, L.L. Zhang, J.H. Ren, Z.Y. Li, et al. The role of sema4D in vasculogenic mimicry formation in non-small cell lung cancer and the underlying mechanisms. Int J Cancer. 2019, 144(9): 2227-2238.

28. J.R. Basile, R.M. Castilho, V.P. Williams and J.S. Gutkind. Semaphorin 4D provides a link between axon guidance processes and tumor-induced angiogenesis. Proc Natl Acad Sci U S A. 2006, 103(24): 9017-22.

29. T. Sawano, F. Watanabe, M. Ishiguchi, N. Doe, T. Furuyama and S. Inagaki. Effect of Sema4D on microglial function in middle cerebral artery occlusion mice. Glia. 2015, 63(12): 2249-59.

30. Y.F. Zhou, Y.N. Li, H.J. Jin, J.H. Wu, Q.W. He, X.X. Wang, et al. Sema4D/PlexinB1 inhibition ameliorates blood-brain barrier damage and improves outcome after stroke in rats. FASEB J. 2018, 32(4): 2181-2196.

31. Y. Zhang, Q. Li, R. Zhuang, Z. Gao, J. Liu, J. Li, et al. Plexin-B1: a potential diagnostic biomarker for glioma and a future target for glioma immunotherapy. J Neuroimmunol. 2012, 252(1-2): 113-7.

32. G. Li, L. Ma, H. Lu, G. Cao, X. Shao, Y. Liu, et al. Transactivation of Met signalling by semaphorin4D in human placenta: implications for the pathogenesis of preeclampsia. J Hypertens. 2018, 36(11): 2215-2225.

33. J.J. Lu, Y.W. Su, C.J. Wang, D.F. Li and L. Zhou. Semaphorin 4D promotes the proliferation and metastasis of bladder cancer by activating the PI3K/AKT pathway. Tumori. 2019, 105(3): 231-242.

34. N. Xu, B. Liu, C. Lian, D.M. Doycheva, Z. Fu, Y. Liu, et al. Long noncoding RNA AC003092.1 promotes temozolomide chemosensitivity through miR-195/TFPI-2 signaling modulation in glioblastoma. Cell Death Dis. 2018, 9(12): 1139.

35. L. Shao, Q. He, Y. Liu, X. Liu, J. Zheng, J. Ma, et al. UPF1 regulates the malignant biological behaviors of glioblastoma cells via enhancing the stability of Linc-00313. Cell Death Dis. 2019, 10(9): 629. 
36. B.J. Reon, J. Anaya, Y. Zhang, J. Mandell, B. Purow, R. Abounader, et al. Expression of IncRNAs in Low-Grade Gliomas and Glioblastoma Multiforme: An In Silico Analysis. PLoS Med. 2016, 13(12): e1002192.

37. J. Fan, J. Zhang, S. Huang and P. Li. IncRNA OSER1-AS1 acts as a ceRNA to promote tumorigenesis in hepatocellular carcinoma by regulating miR-372-3p/Rab23 axis. Biochem Biophys Res Commun. 2019, doi: 10.1016/j.bbrc.2019.10.105.

38. Y. Tay, J. Rinn and P.P. Pandolfi. The multilayered complexity of ceRNA crosstalk and competition. Nature. 2014, 505(7483): 344-52.

39. R. Wang, S. Zhang, X. Chen, N. Li, J. Li, R. Jia, et al. CircNT5E Acts as a Sponge of miR-422a to Promote Glioblastoma Tumorigenesis. Cancer Res. 2018, 78(17): 4812-4825.

40. Q. Wang, J. Cai, C. Fang, C. Yang, J. Zhou, Y. Tan, et al. Mesenchymal glioblastoma constitutes a major ceRNA signature in the TGF-beta pathway. Theranostics. 2018, 8(17): 4733-4749.

\section{Figures}




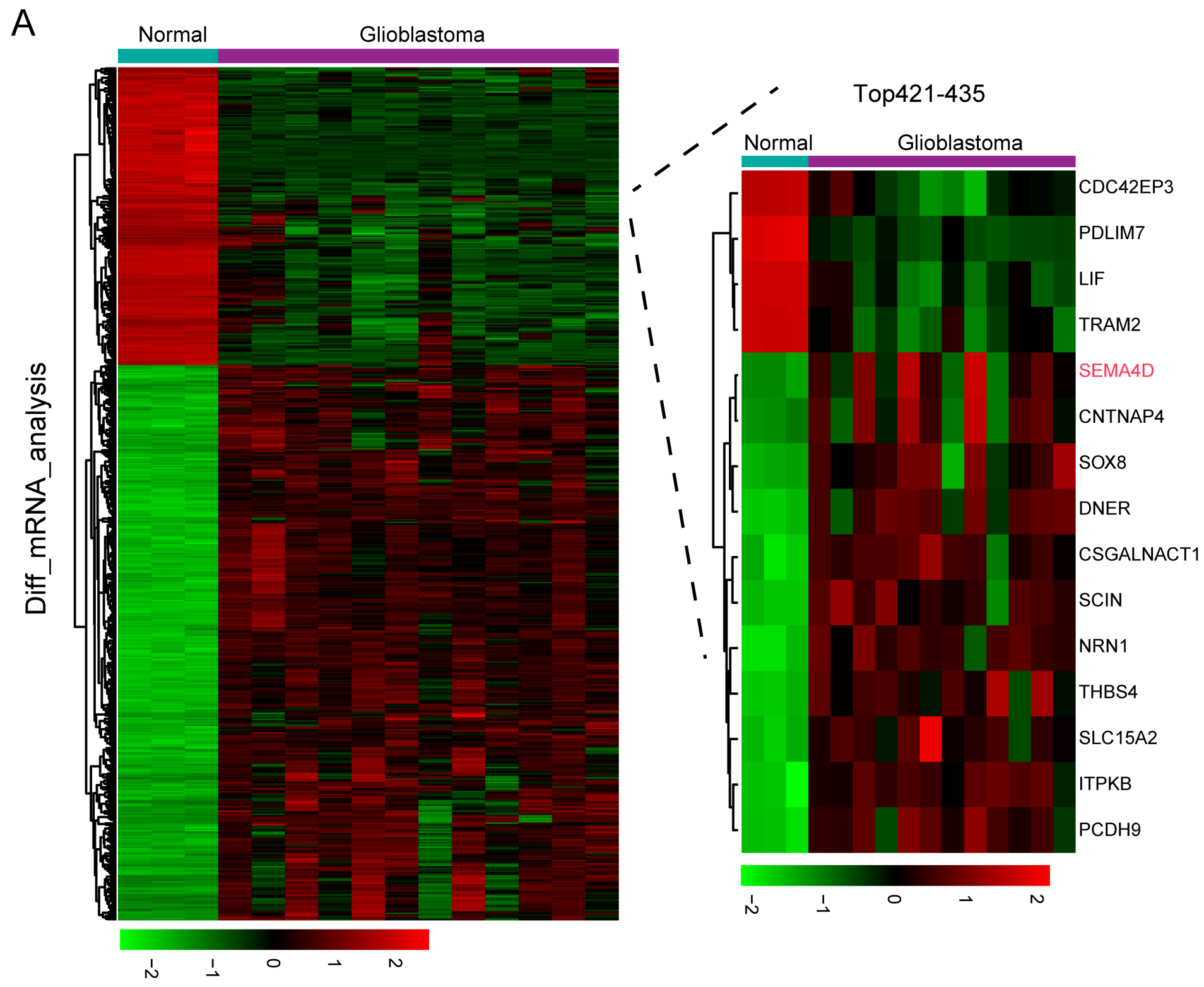

\section{Figure 1}

The differentially expressed genes between GBM and normal astrocytes. (A) All of the differentially expressed genes. (B) Top 421-435 differentially expressed genes. 


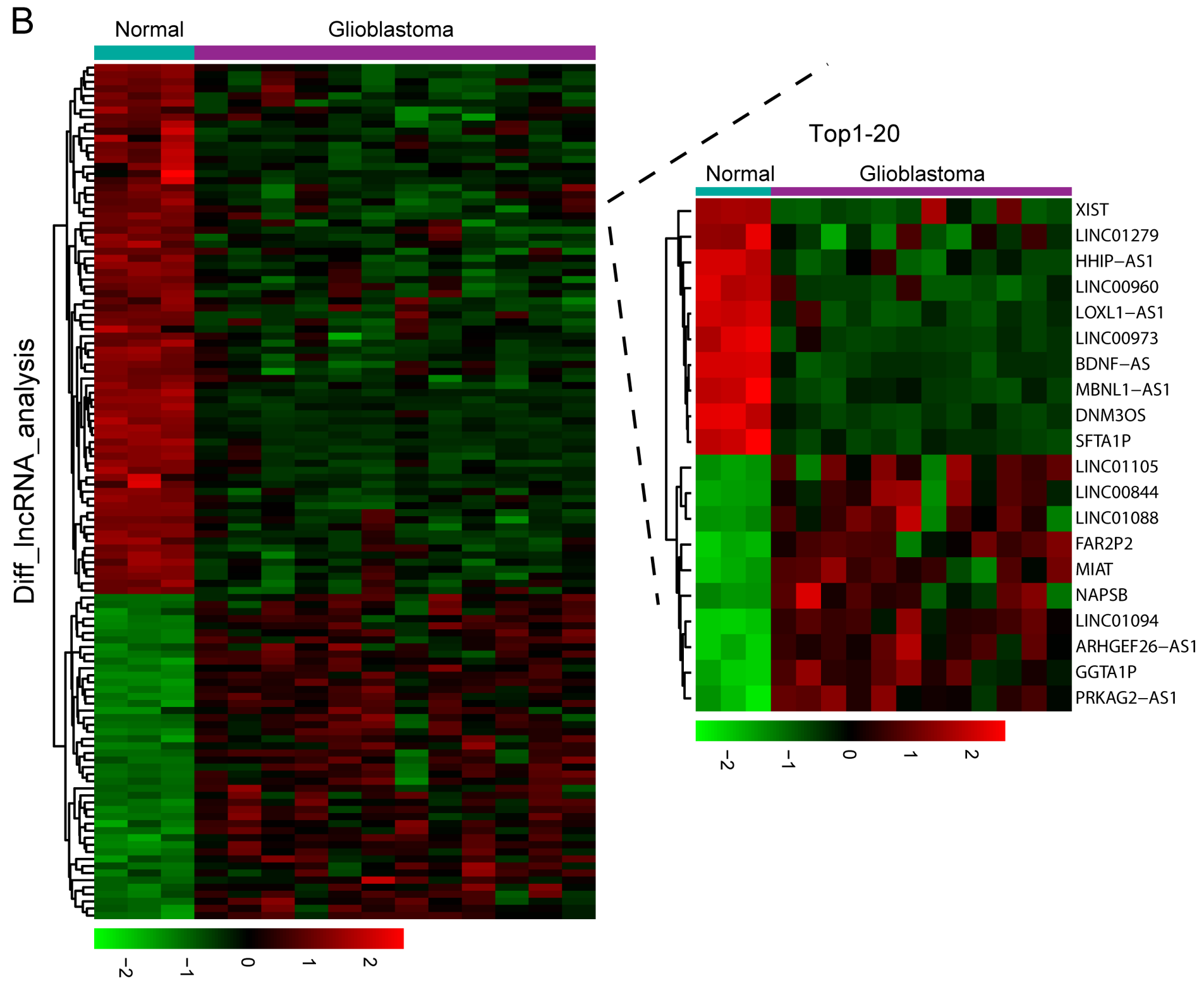

Figure 2

The differentially expressed IncRNAs between GBM and normal astrocytes. (A) All of the differentially expressed IncRNAs. (B) Top 1-20 differentially expressed IncRNAs. 
A

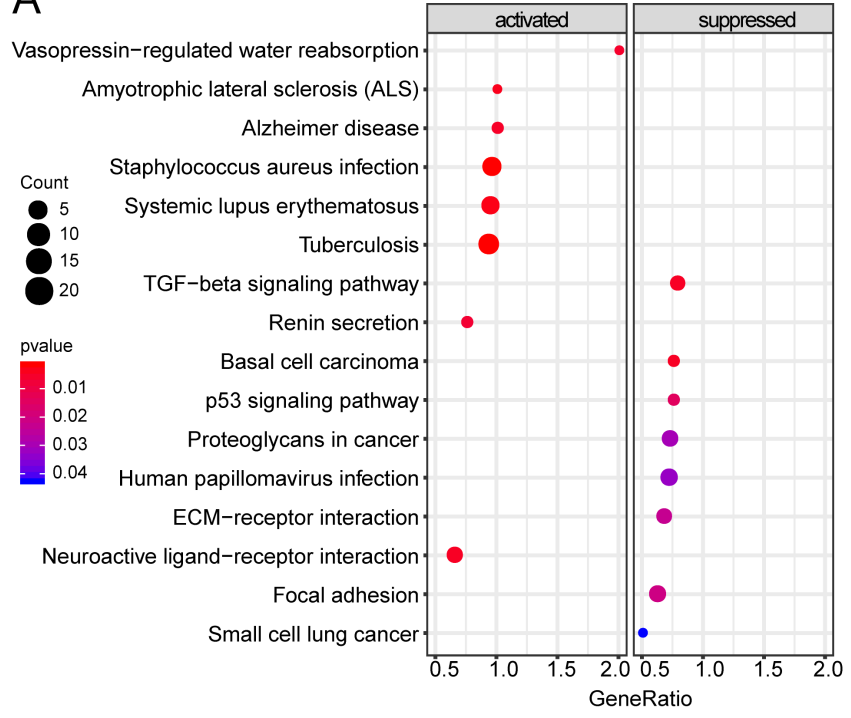

C

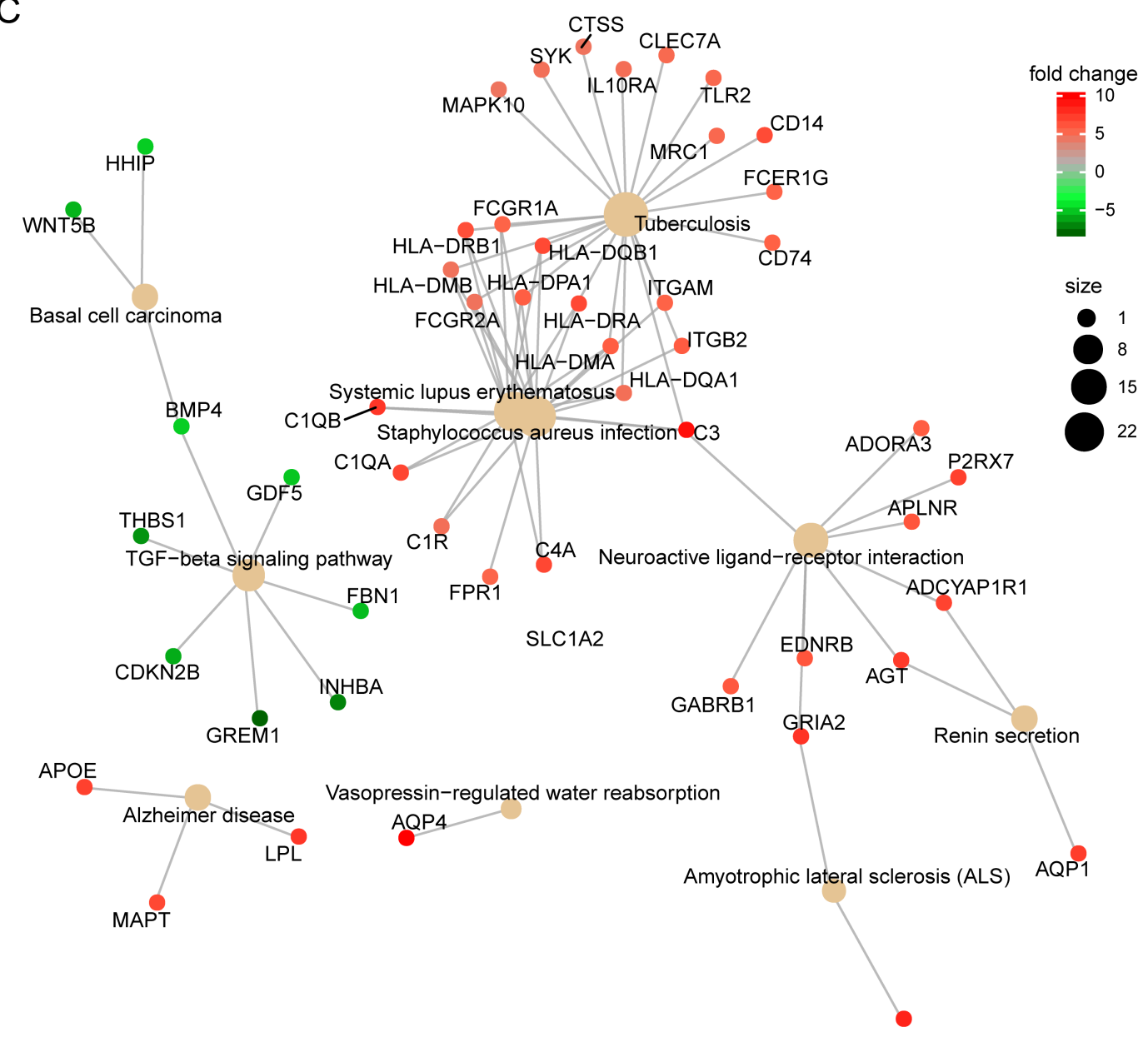

\section{Figure 3}

The signaling pathway enrichment was analyzed based on gseKEGG. (A) The enriched signaling pathways were presented by dotpolt. (B) The enrichment map showed the relevance between the enriched signaling pathways. (C) Connect plot revealed the connection between significantly activated or suppressed signaling pathways and differentially expressed genes. 


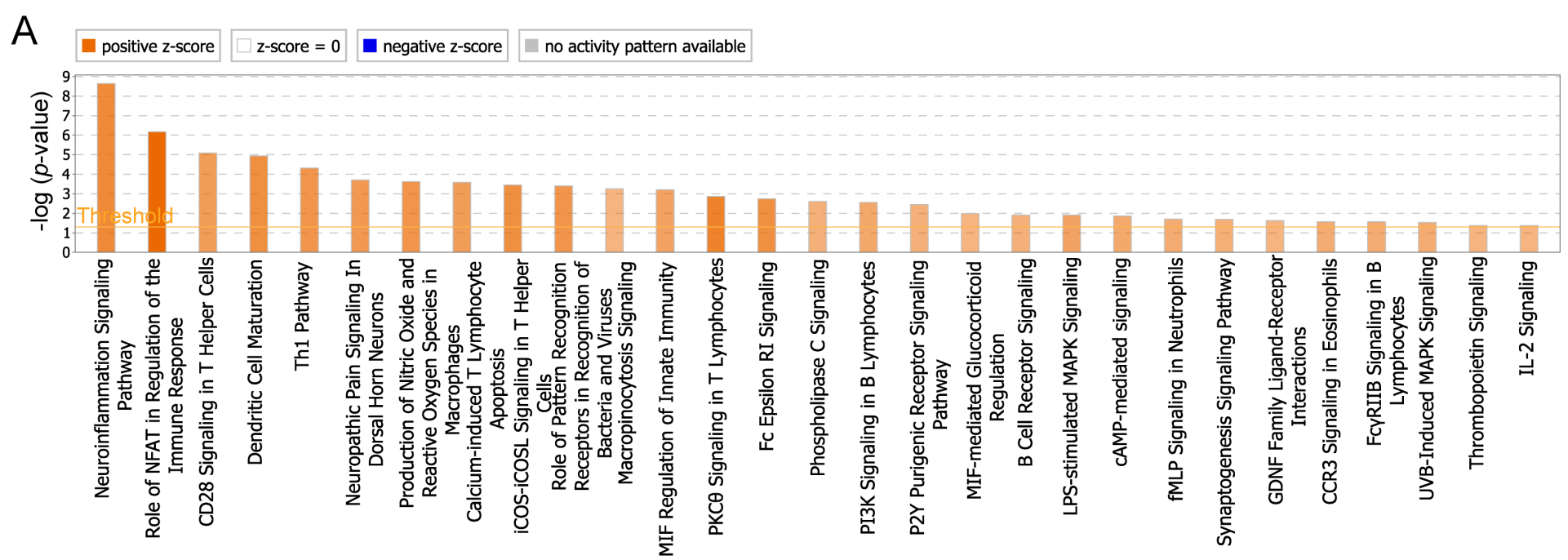

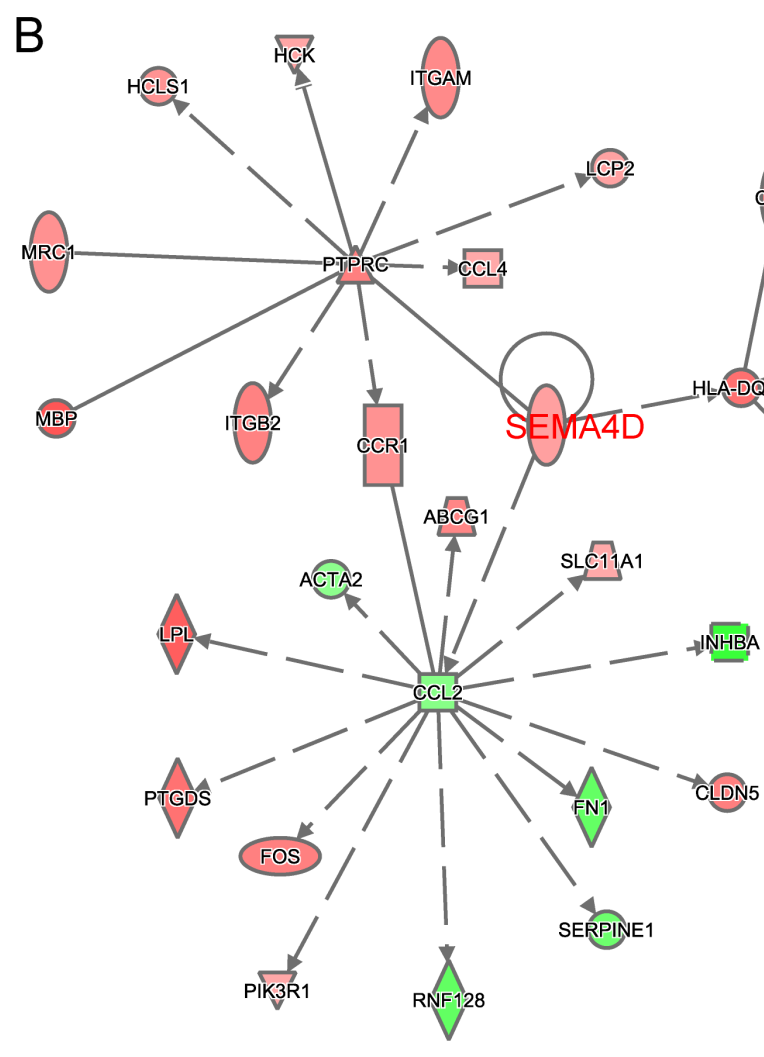

mRNA-mRNA-correlation

\section{C}
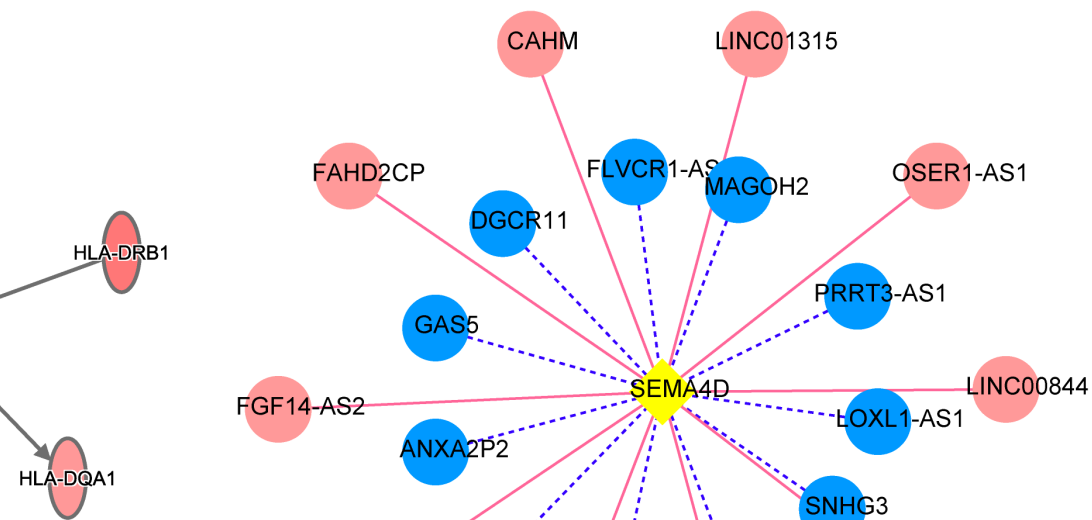

Dorotion

$\triangle$ Enzyme
$\square$ G-protein Coupled Receptor

Growth Factor

$\vec{\nabla}$ Kinase

Other

$\triangle$ Phosphatase

$\bigcirc_{1}$ Transcription Regulator

O Transmembrane Receptor

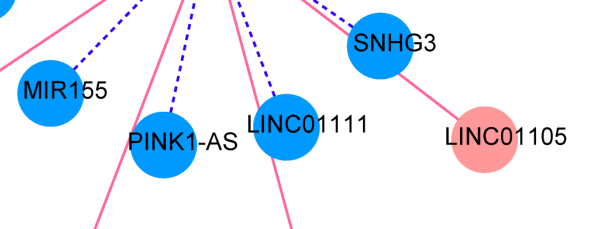

SOX2-OT PRKAG2-AS1

cutoff $>0.7 \quad p$ val $<0.05$

mRNA-IncRNA-correlation

\section{Figure 4}

The canonical pathway and correlation analysis were performed. (A) Significant enrichment of differentially expressed genes in canonical pathways. Orange indicates that the pathway is activated (zscore $>0$ ), while blue indicates that the pathway is inhibited $(z$-score $<0)$. (B) The correlation between differentials expressed genes. Pink represents upregulation of mRNAs, and the cyan represents downregulation of mRNAs. (C) Co-expression network between SEMA4D and differentially expressed IncRNAs. Pink circles represent upregulation of IncRNAs, and the blue circles represent downregulation of IncRNAs. 


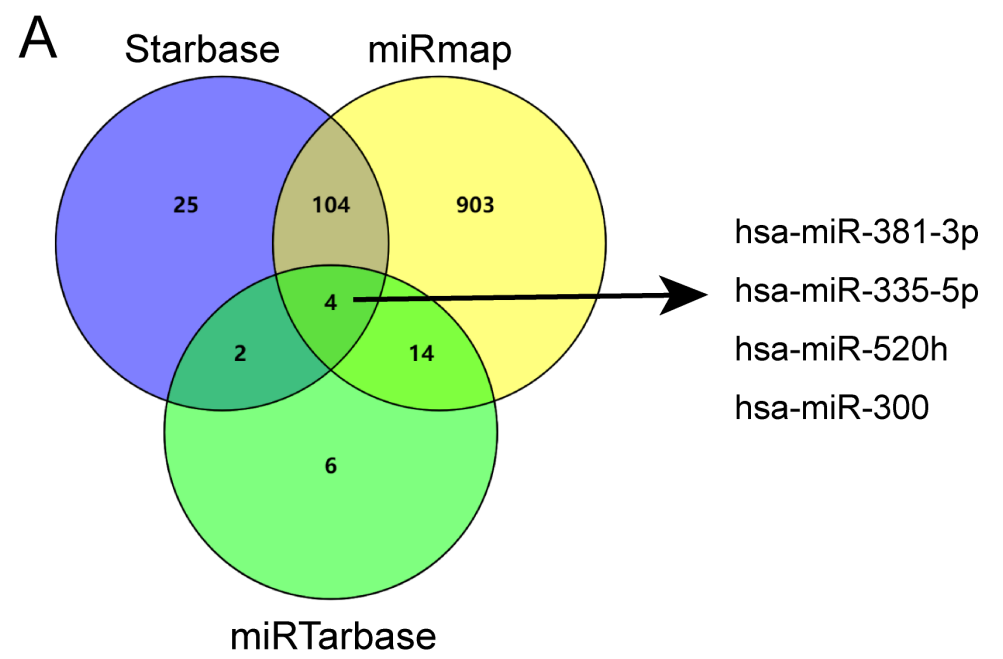

B

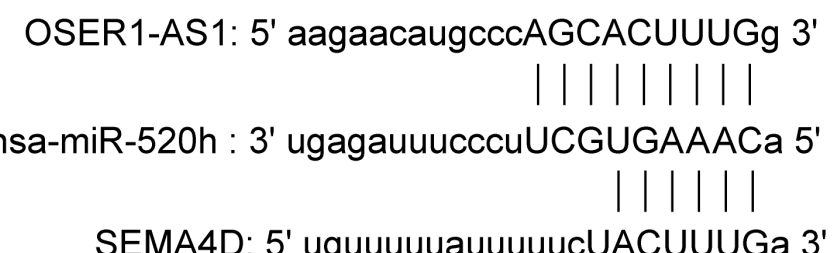

Figure 5

miRNAs targeting SEMA4D were screened and predicted possible ceRNA mechanisms. (A) miRNAs targeting SEMA4D were screened by Venny based on Starbase, miRmap and miRTarbase databases. (B) The target sites between IncRNA OSER1-AS1 and miR-520h as well as miR-520h and SEMA4D were predicted.

\section{Supplementary Files}

This is a list of supplementary files associated with this preprint. Click to download.

- Supplementarytable3.xlsx

- Supplementarytable2.xlsx

- Supplementarytable1.xlsx 